\title{
Refining Stimulus Parameters in Assessing Infant Speech Perception Using Visual Reinforcement Infant Speech Discrimination in Infants with and without Hearing Loss: Presentation Level
}

DOI: $10.3766 /$ jaaa.17061

\author{
Kristin M. Uhler* \\ René H. Gifford $\dagger$ \\ Jeri E. Forster \\ Melinda Anderson§ \\ Elyse Tierney§ \\ Stacy D. Claycomb** \\ Lynne A. Werner $\dagger \dagger$
}

\section{INTRODUCTION}

$\mathrm{U}$ niversal newborn hearing screening has led to a decrease in the average age at identification and treatment of hearing loss (HL) (Harrison and Roush, 1996; Moeller, 2000; Holte et al, 2012; Uhler et al, 2016). Despite identification at earlier ages, there continue to be gaps in language outcomes between children with HL and their peers with normal hearing (NH). Studies that have followed children with HL from infancy through early school age have identified two important predictors of outcome: amount of hearing aid use (Moeller et al, 2009; Walker et al, 2013; Walker et al, 2015) and the quality of hearing aid fittings (McCreery et al, 2013; 2015), suggesting that the quantity and quality of speech input are critical variables. Moreover, a higher aided speech intelligibility index (SII) is associated with better later language in preschool children (Tomblin et al, 2014; 2015) and improved word recognition in school-aged children (Stiles et al, 2012).

The current clinical best practice of performing realear measures only verifies hearing aid output in the ear canal. This measure alone cannot ensure that amplification is providing infants and young toddlers with the information needed to discriminate between speech sounds-a prerequisite for learning spoken language (Tsao et al, 2004; Tomblin et al, 2014; 2015). A clinically useful tool for directly assessing speech discrimination in infancy could help to determine that infants and toddlers with HL are fitted appropriately. Currently, the most commonly used tools for assessing speech perception in infants and toddlers are parent questionnaires (Uhler and Gifford, 2014), which are not objective measures of speech discrimination.

A clinically useful tool capable of assessing speech discrimination in infancy has been available since 1989 (Gravel, 1989). Visual Reinforcement Infant Speech Discrimination (VRISD) uses a conditioned head turn task, similar to visual reinforcement audiometry (VRA). However, rather than infants being conditioned to the presence of a tone or speech, in VRISD, the infant is conditioned to turn his/her head to a change in stimulus. VRISD has been primarily used in research laboratories, despite its relative familiarity in clinical audiology as a derivative of VRA. A lack of clinical guidelines and normative data may be one reason that VRISD has not seen widespread clinical adoption.

Establishing appropriate presentation levels is one prerequisite for the clinical application of VRISD. Nozza

*University of Colorado Denver School of Medicine, Children's Hospital Colorado, Aurora, C0; †Vanderbilt University School of Medicine, Nashville, TN; $\$$ Rocky Mountain Mental Illness, Research, Education and Clinical Center, Denver VA Medical Center and University of Colorado Denver School of Medicine, Aurora, CO; §University of Colorado Denver School of Medicine, Aurora, C0; ${ }^{*}$ University of Colorado Health, Aurora, CO; $\dagger$ †University of Washington, Seattle, WA

Corresponding author: Kristin M. Uhler, Aurora, CO 80045; Email: kristin.uhler@ucdenver.edu

This project was funded by the American Academy of Audiology/American Academy of Audiology Foundation Research Grants Program and grant funding from NIH NIDCD DC013583 to KU.

Parts of this work were presented at the ARC in Indianapolis, IN, April 2017. 
and colleagues showed that the relationship between speech discrimination performance in VRISD and presentation level differs between infants and adults (Nozza and Wilson, 1984; Nozza, 1987; Nozza et al, 1991; Nozza, 2000). They found that NH infants between 6 and 8 months of age required a higher presentation level in quiet and a more favorable signal-to-noise ratio than $\mathrm{NH}$ adults to attain maximum performance. Furthermore, Nozza (2000) reported that the lowest sensation level (i.e., level relative to individual detection threshold) at which infants could discriminate between $/ \mathrm{ba} /$ and $/ \mathrm{da} /$ was 20-25 dB compared with 10-15 dB for adults. These findings suggest that the typical procedure of assessing speech perception in infants at the same intensity level as used for adults may underestimate infant speech perception abilities (Eilers et al, 1977; 1981; Martinez et al, 2008; Fredrickson, 2010; Uhler et al, 2011).

In a recent VRISD study, Uhler et al (2015) showed that the level at which $\mathrm{NH}$ infants successfully discriminated /a-i/ and /ba-da/ ranged from 35 to $70 \mathrm{~dB}$ SL. NH infants needed a higher presentation level to discriminate /ba-da/ than /a-i/ and consistent with the results of Nozza (1987), 29\% were unable to discriminate /ba-da/ at the highest presentation level (70 dBA). $\mathrm{NH}$ infants who did not reach criterion on one or both contrasts did not significantly differ in age, gender, or audiometric thresholds from the infants who reached criterion. Thus, there is some inherent variability in the mastery of/ba-da/ discrimination, even for infants with $\mathrm{NH}$, making it all the more important to directly evaluate infants with HL.

The goal of the current study was to extend the previous work with $\mathrm{NH}$ infants to infants and toddlers with HL. We have four primary research questions, which are approached with a goal of clinical utility and ecological validity. First, what is the presentation level at which most infants reach criterion for speech discrimination? Second, is the criterion presentation level different for infants with HL than infants with NH? Third, is there a difference in criterion presentation level for the /a-i/ contrast compared with /ba-da/? Finally, to assess whether VRISD tells us something about the quality of sound input beyond that provided by currently available measures, we investigated the relationship between aided SII and speech discrimination for infants who use hearing aids (HAs).

\section{METHODS}

\section{Participants}

Data were collected for 43 children aged 7-28 months $(M=13.91, \mathrm{SD}=5.81)$. The data for the 21 children with $\mathrm{NH}$ were previously reported (Uhler et al, 2015). The 22 infants with bilateral sensorineural HL had losses ranging from mild to profound. Two subjects were excluded from participation after consenting. One was a 15-monthold male with bilateral cochlear implants (CIs) who could not be conditioned for the hearing test. The second was an 11-month-old male with severe to profound HL with bilateral HAs who could not be conditioned at the second VRISD session. Of the 20 remaining subjects, 17 used bilateral HAs and three used bilateral CIs. There were 11 males and nine females. The age at hearing aid fitting, both for infants using HAs and for those who transitioned to CI, ranged from 2 to 8 months $(M=$ 3 months; SD $=1.98$ months). Demographic information for participants from this study as well as the NH listeners (from Uhler et al, 2015) appears in Table 1.

The criteria for inclusion were (a) no evidence of significant developmental delays or secondary disabilities per parent report or as indicated in the electronic medical record, (b) demonstrated conditioned head turn in VRA, (c) normal tympanometry on the day of testing or patent pressure equalization tubes, (d) enrollment in early intervention, (e) use of HAs and/or CIs daily per parent report, and (f) either English or Spanish as the primary language spoken in the home. Criteria for exclusion were (a) a history of untreated chronic middle ear infections paired with abnormal tympanometric findings on the day of testing and (b) auditory neuropathy.

Table 1. Subject Characteristics

\begin{tabular}{|c|c|c|c|c|}
\hline & $\mathrm{NLN}=21$ & $\mathrm{HLN}=20$ & Statistical Test & $p$ Value \\
\hline Age in months & $10.3(2.9)$ & $13.9(5.8)$ & $t=2.51$ & 0.02 \\
\hline Male & $8(38 \%)$ & $11(55 \%)$ & $X^{2}=1.18$ & 0.28 \\
\hline Randomization $=1$ & $12(57 \%)$ & $8(40 \%)$ & $x^{2}=1.20$ & 0.27 \\
\hline $\begin{array}{l}\text { Better Ear, unaided (for } \mathrm{NL} \text { ) } \\
\text { and aided } 4 \mathrm{~F} \text { PTA for } \mathrm{HL} \\
\text { listeners }(\mathrm{dB}) \text {, mean }(\mathrm{SD}) \mathrm{dB} \mathrm{HL}\end{array}$ & Unaided 13.33 (2.55) & $\begin{array}{l}\text { Aided } 27.25(13.4) \\
\text { Unaided } 50.71(95 \% \text { Cl 39.75-61.66) }\end{array}$ & $t=4.58^{\star}$ & 0.0002 \\
\hline Threshold detection of /a/ dB A & $5.71(5.07)$ & $19.75(10.19)$ & $t=5.54^{*}$ & $<0.0001$ \\
\hline
\end{tabular}


All participants used their own HAs or CIs during aided testing. The managing audiologists confirmed that all participants' HAs were programmed using Desired Sensation Level v5.0 (DSL; Scollie et al, 2005). Before laboratory testing, all devices were evaluated to assure proper function. An electroacoustic test box measure was completed to assess hearing aid function. In addition, the HA output was measured either using measured or simulated real ear coupler differences. The SII was automatically calculated at 55 and $65 \mathrm{~dB}$ SPL for all but one participant whose HAs were measured only at $65 \mathrm{~dB}$ SPL, using the Audioscan Verifit. CI processor function was verified both by listening check and ensuring that CI-aided detection thresholds were less than $30 \mathrm{~dB}$ HL from 500 to $4000 \mathrm{~Hz}$.

Aided and unaided pure-tone and speech awareness thresholds were assessed using VRA in sound field for each HA user. Children with CI were similarly tested, but only with their CI because they had insufficient residual hearing to provide unaided thresholds. The hearing assessment served to verify hearing sensitivity and to confirm the child's ability to successfully complete a conditioned head turn task.

Both the Colorado Multiple Institutional Review Board and the Vanderbilt Institutional Review Board approved this project. Consent was obtained from parents/guardians before beginning the research project. Parents were provided with compensation for their child's participation.

\section{Stimuli}

Four stimuli were used: /a/, /i/, /ba/, and /da/. The two contrasts used for the experiment were /a-i/ and /ba-da/. These contrasts represent different levels of difficulty, with the vowel contrast (/a-i/) being easiest and the place of articulation contrast (/ba-da/) being most difficult for both $\mathrm{NH}$ children and children with sensorineural HL (Boothroyd, 1984; Martinez et al, 2008; Uhler et al, 2015). The stimuli were natural speech tokens produced by a female speaker, and adult listeners in the laboratory verified that the stimuli sounded natural. Stimuli were then digitized using a 16-bit analogto-digital converter (AD Instruments Power Laboratory/ $16 \mathrm{SP})$ at $40 \mathrm{kHz}$ and edited using Goldwave Inc. (St. John's, NL, Canada). The stimuli were down sampled to $22050 \mathrm{~Hz}$ and edited to $500 \mathrm{msec}$ duration. The /ba/ and /da/ stimuli used for testing were constructed by appending the /a/ used in the vowel discrimination task to each of the consonants to maintain consistency of the vowel sound. For consonant-vowel stimuli, the duration of the consonant was $100 \mathrm{msec}$, and the duration of the vowel was $400 \mathrm{msec}$. During testing, stimuli were presented with 1,200 msec interstimulus interval. Stimuli were root mean squared (RMS) equalized and presented at either 50, 60, and/or $70 \mathrm{dBA}$. Figure 1 shows a spectrogram of each stimulus.

\section{Testing Protocol}

Testing was completed in a double-walled sound booth. The digitized speech stimuli were routed to an audiometer for presentation in the sound field.

For the infants with HL, two sessions were required to complete the protocol. The first session consisted of the case history (including amount of daily device use), unaided and/or aided hearing test, and if time allowed, an aided threshold search for /a/. The second visit consisted of the aided threshold search for $/ \mathrm{a} /$ if not completed at the first visit and the VRISD assessment protocol.

During VRISD testing, one of the speech sounds for the contrast pair served as the background stimulus,

\section{A \\ Vowel contrast}
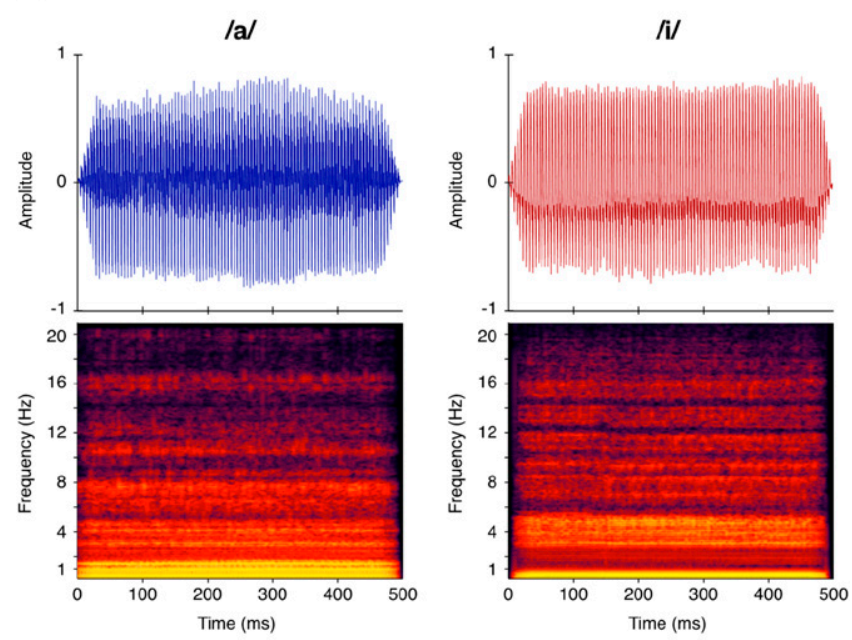

B

Consonant contrast
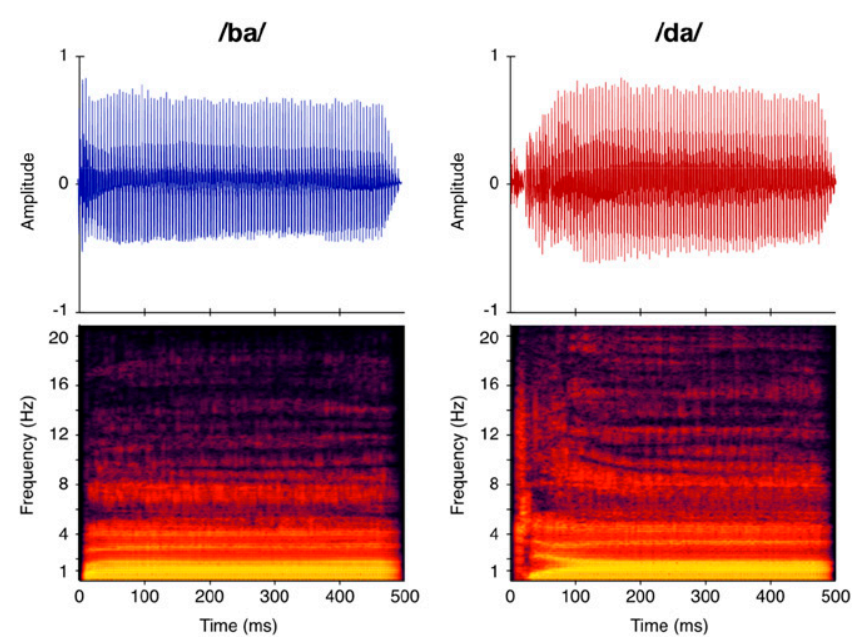

Figure 1. Waveform and corresponding spectrogram of target and background stimuli. Panel (A) is the vowel contrast /a-i/ and (B) is the consonant contrast/ba-da/. For each panel, the top illustrates the amplitude waveform of the stimuli and the bottom illustrates the frequency spectrogram of the stimuli. As can be seen there is less spectral difference for the consonant contrast. 
while the other served as the target. The background stimulus was presented repeatedly between trials. The infant learned to respond when the target stimulus was presented. The member of the pair serving as the target stimulus was counterbalanced across subjects. The order of vowel and consonant testing was also counterbalanced across subjects.

The infant was accompanied by his/her caregiver into the sound booth for the VRISD assessment. The child was either seated on the caregiver's lap or in a high chair in the center of the room. The background stimulus was on when the child entered the room. The speaker and visual reinforcement video screen were placed at $90^{\circ}$ to the right of the child's midline. An assistant who centered the infant's gaze was positioned in front of the child slightly to the left. The caretaker and the assistant listened to music through supra-aural headphones to prevent them from hearing the sounds presented to the child and inadvertently reinforcing or alerting the child to a contrast stimulus.

There were two evaluators for this study and both followed the same methods for assessing VRISD. The evaluator, seated outside the sound booth in a test room observed the child through a window. The evaluator could not hear the stimuli. The evaluator initiated trials by pressing a button once the child's attention was directed toward midline. Fifty percent of trials were nochange trials and $50 \%$ were change trials; the computer program randomly determined which trial type was presented. The evaluator was blind to trial type. If the trial was a no-change trial, the background sound was presented three times. If the trial was a change trial, the target sound was presented three times. At the end of the trial, the background sound continued. The evaluator indicated whether the child executed a head turn toward the speaker by pressing another button. The VRISD software determined if the child's head turn was a correct response to a change trial or a false positive to a no-change trial. Correct responses were rewarded by automatic presentation of a visual reinforcer, an animated video. Fifteen trials were administered during each contrast assessment.

Once the 15 trials were completed, the evaluator calculated the proportion correct while the child remained in the test booth. If the child achieved at least 0.75 proportion correct for the contrast at $50 \mathrm{dBA}$, then testing for the first contrast was complete and testing for the second contrast was initiated (Nozza, 2000; Uhler et al, 2015). The rationale was that a child who can successfully discriminate at a low level would also be able to discriminate at higher levels (McArdle and HnathChisolm, 2009). However, if the child did not reach criterion at $50 \mathrm{dBA}$, then the level was increased to $70 \mathrm{dBA}$ and testing resumed. Once 15 trials at $70 \mathrm{dBA}$ were completed, the level was reduced to $60 \mathrm{dBA}$ and 15 trials were completed at that level, regardless of performance at $70 \mathrm{dBA}$. This process was repeated for the other contrast. Therefore, children who did not reach criterion at
$50 \mathrm{dBA}$ for either contrast completed a total of six conditions (/a-i/ and /ba-da/ at 50, 60, and $70 \mathrm{dBA})$. In each session, testing continued until all conditions were completed or until the child was too fussy or tired to continue.

\section{Statistical Analysis}

To achieve an unbiased estimate of sensitivity, we converted the scores from proportion correct to $p(C)_{\max }$, the proportion correct achieved by an unbiased observer with a given sensitivity or d' (Macmillan and Creelman, 2005). The advantage of $p(C)_{\max }$ is that it eliminates the effect of response bias, but expresses sensitivity on a widely understood scale (i.e., proportion correct). For one subject the raw data could not be recovered, and the recorded proportion of correct responses were used in the analysis. The criterion for mastery of a contrast was a $p(C)_{\max } \geq 0.75$.

\section{RESULTS}

$\mathrm{F}$ our sets of analyses were carried out to address our primary research aims. First, we considered the proportion of infants with HL who were able to discriminate each contrast at each presentation level. Second, we compared the proportion of infants with HL and the proportion of infants with $\mathrm{NH}$ who reached criterion at each presentation level for each of the contrasts. Third, we analyzed the data using survival analysis to determine the estimated probability of discrimination at each presentation level for each contrast for infants with $\mathrm{NH}$ and infants with HL. Finally, we examined the relationships between aided SII and performance on VRISD for each contrast at $50 \mathrm{dBA}$.

\section{Effect of Presentation Level on Speech Discrimination Performance}

Table 2 lists the proportion of infants who reached criterion on each contrast in each group. Among infants with $\mathrm{HL}, 50 \%$ reached criterion on $/ \mathrm{a}-\mathrm{i} /$ at $50 \mathrm{dBA}$ and $95 \%$ reached criterion on /a-i/ at some presentation level. Only $50 \%$ of the infants with HL reached criterion on /ba-da/ at any presentation level. Thus, the presentation levels used here were sufficient to allow nearly all infants with HL to demonstrate discrimination of /a-i/, but not /ba-da/.

\section{Differences between Infants with HL and Infants with NH}

Although a higher proportion of infants with $\mathrm{NH}$ than of infants with HL reached criterion at $50 \mathrm{dBA}$ for /a-i/, the difference was not significant $\left(X^{2}=0.59, p=0.44\right)$. Moreover, $95 \%$ of infants with HL and $86 \%$ of infants with $\mathrm{NH}$ reached criterion on /a-i/ at some presentation level. Those results suggest that the effect of presentation level on speech discrimination is similar for the two 
Table 2. Infant Performance by Contrast and Intensity Level

\begin{tabular}{|c|c|c|c|c|c|}
\hline \multirow[b]{2}{*}{ Contrast } & \multirow[b]{2}{*}{ Level (dBA) } & \multicolumn{2}{|c|}{ Infants with $\mathrm{HL}$} & \multicolumn{2}{|c|}{ Infants with $\mathrm{NH}$} \\
\hline & & Number of Subjects & $\%$ of Subjects & Number of Subjects & $\%$ of Subjects \\
\hline \multirow[t]{3}{*}{$\overline{\mathrm{a}-\mathrm{i} /}$} & 50 & 10 & 50 & 12 & 57 \\
\hline & 60 & 7 & 35 & 2 & 10 \\
\hline & 70 & 2 & 10 & 4 & 19 \\
\hline \multicolumn{2}{|c|}{ Did not reach } & 1 & 5 & 3 & 14 \\
\hline \multirow[t]{3}{*}{ /ba-da/ } & 50 & 5 & 25 & 9 & 43 \\
\hline & 60 & 3 & 15 & 2 & 10 \\
\hline & 70 & 2 & 15 & 4 & 19 \\
\hline \multicolumn{2}{|c|}{ Did not reach } & 10 & 50 & 6 & 29 \\
\hline
\end{tabular}

Note: For each contrast, the lowest level at which criterion was reached was determined. If a subject reached criterion at $50 \mathrm{dBA}$, then no additional levels were assessed. However, if criterion was not reached at $50 \mathrm{dBA}$, the subject was tested at all three levels for that contrast. Thus, the subjects who reached criterion at $50 \mathrm{dBA}$ are not included in the totals at 60 or 70 dBA. Percentages were rounded to the nearest whole number, therefore, do not add up to $100 \%$.

groups of infants for /a-i/. For /ba-da/, the proportion of infants reaching criterion at $50 \mathrm{dBA}$ did not differ between groups $\left(X^{2}=0.59, p=0.37\right)$. While $71 \%$ of infants with $\mathrm{NH}$ reached criterion at some presentation level for /ba-da/, only $50 \%$ of infants with HL did so; however, that difference was not statistically significant $\left(X^{2}=\right.$ $0.81, p=0.37)$. Finally, the proportion of infants with HL who achieved criterion on /a-i/ at some presentation level was higher than that on $/ \mathrm{ba}-\mathrm{da} /(p=0.004)$, but the difference between contrasts was not significant for the infants with $\mathrm{NH}(p=0.45)$. Those results suggest that /ba-da/ was a relatively more difficult contrast for infants with HL than for infants with $\mathrm{NH}$.

\section{Estimated Probability of Discrimination at Each Presentation Level}

To estimate the probability of reaching criterion on a contrast as a function of presentation level, we used parametric survival analysis models. Survival analysis is a set of methods used for analyzing event occurrence data where the outcome variable has two parts: one is event status and the other is the time to event. In the context of the current study, the event of interest was reaching criterion on VRISD, and the second dimension was presentation level (dBA) rather than time. Simply stated, if an infant did not discriminate at $50 \mathrm{dBA}$ then that observation was considered to have "survived", and the infant continued testing at both 60 and $70 \mathrm{dBA}$. The advantage of survival analysis over other approaches is that it uses all of the available data to estimate the probability of success at each level, while controlling in this case for age, sex, and test order of the contrasts.

Figure 2 plots estimated probability curves from the survival models for the two groups of infants for each of the speech contrasts. The point at which a curve crosses the 0.75 success rate is an estimate of the presentation level required for $75 \%$ of infants to reach criterion for a contrast. As can be seen in the figure, the presentation level for /a-i/ at which infants with $\mathrm{NH}$ are estimated to reach criterion is
$63 \mathrm{dBA}$, while for infants with $\mathrm{HL}$, the level is $56 \mathrm{dBA}$. For the /ba-da/ contrast, both groups of infants are less likely to reach criterion at the levels used in this study than for the /a-i/ contrast. Given the large number of infants who were not able to discriminate/ba-da/, it is not possible to estimate the presentation level where the majority reach criterion. Statistical comparisons between the curves indicated no differences between groups for the estimated level at which criterion is met for the $/ \mathrm{a}-\mathrm{i} /$ contrast $(\beta=0.032, p=0.76)$ or the /ba-da/ contrast ( $\beta=-0.23, p=0.24$ ).

We asked whether infants who reached criterion on /a-i/ $(\mathrm{n}=37)$ were more likely to also reach criterion on /ba-da/ compared with infants who did not reach criterion on $/ \mathrm{a}-\mathrm{i} /(\mathrm{n}=4)$. A Fisher's exact test showed that there was no significant relationship ( $p=0.64$ ) between criterion discrimination abilities for the two pairs of contrasts.

\section{Relationship between Aided SII and Speech Discrimination Performance}

For infants using HAs ( $\mathrm{n}=17$ ), we examined the relationships between performance on VRISD, aided SII, and the high-frequency pure-tone average (HFPTA) using Wilcoxon rank-sum tests. The VRISD data at $50 \mathrm{dBA}$ were used because all infants were tested at this level. Aided SII is a measure of speech audibility and was obtained using measured or simulated real ear measurements. The aided SII of infants who successfully discriminated /a-i/ (median $=0.81,95 \% \mathrm{CI}$ $[0.57,0.96])$ was higher than that of infants who were unsuccessful at discrimination of /a-i/ (median $=0.59$, 95\% CI $[0.23,0.83] ; p=0.02)$. The HFPTA of infants who successfully discriminated /a-i/ (median $=22$, $95 \%$ CI $[10,30])$ was lower than that of infants who were unsuccessful at discrimination of /a-i/ (median $=33,95 \%$ CI $[15,74] ; p=0.03)$. The same relationships were not observed for /ba-da/. Aided SII of infants who successfully discriminated $/ \mathrm{ba}-\mathrm{da} /($ median $=0.49,95 \% \mathrm{CI}[0.63,0.96])$ did not differ from that of infants who were unsuccessful at discrimination of $/ \mathrm{ba}-\mathrm{da} /($ median $=0.66,95 \%$ CI $[0.23$, 


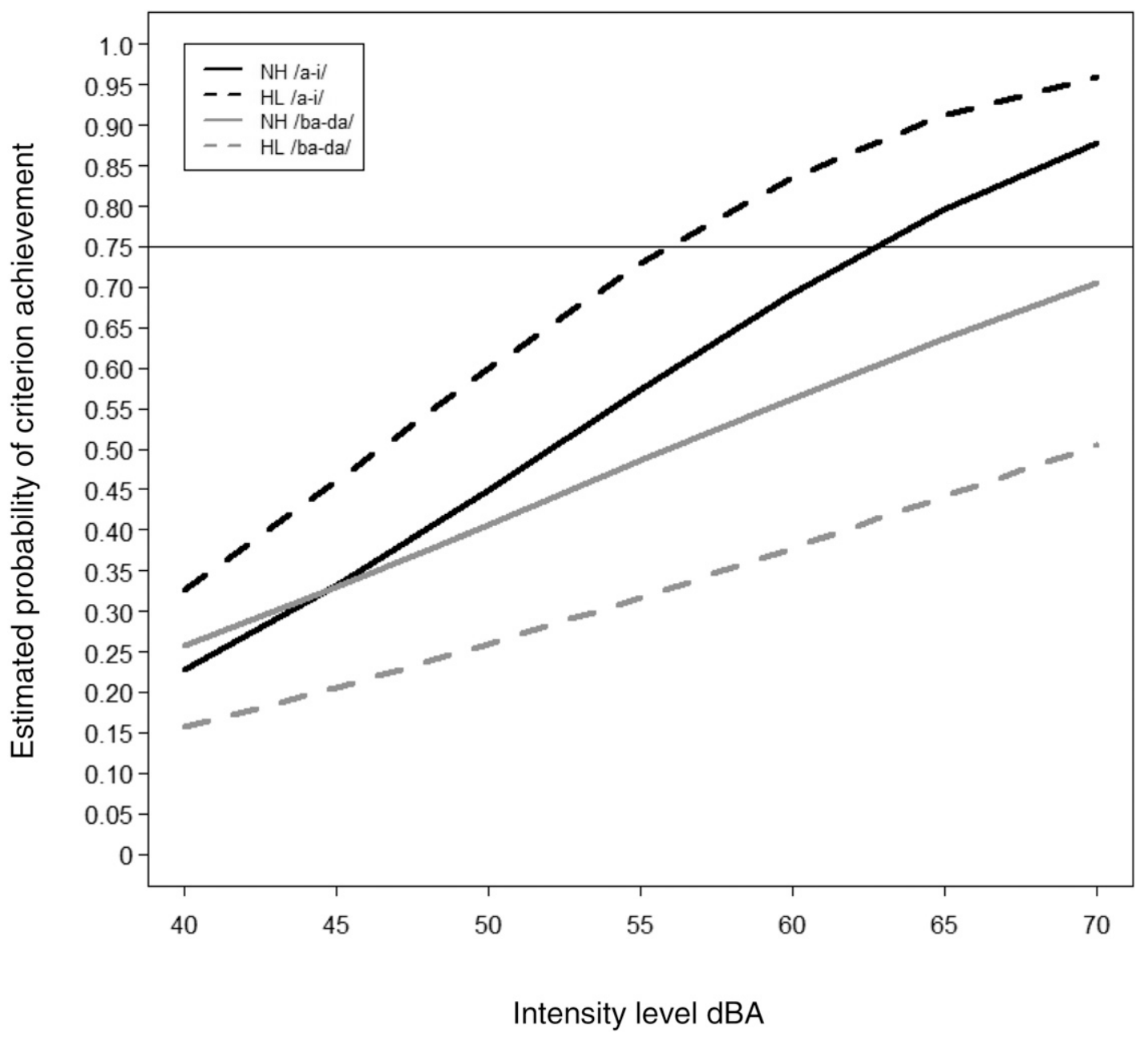

Figure 2. Estimated probability of infant with and without HL reaching criterion based on the survival statistic for the /a-i/ and /ba-da/ contrasts. Performance below $50 \mathrm{dBA}$ is left censored to infer performance. Performance pattern across presentation levels for the participants while controlling for age, sex, and the randomized order of the contrasts. The presentation level at which infants with normal hearing are estimated to reach criterion for the /a-i/contrast is $63 \mathrm{dBA}$. For children with HL, the level is $56 \mathrm{dBA}$. For the/ba-da/ contrast, it is not possible to infer the levels at which criterion would be reached because levels greater than $70 \mathrm{dBA}$ were not examined.

$0.93] ; p=0.20$ ). Similarly, HFPTA of infants who successfully discriminated /ba-da/ (median $=26,95 \%$ CI $[15,38])$ did not differ from that of infants who were unsuccessful at discrimination of $/ \mathrm{ba}$-da/ (median $=26.5,95 \%$ CI $[10$, $74] ; p=0.78$ ). Thus, while aided SII and HFPTA were predictive of /a-i/ discrimination, those measures were not predictive of /ba-da/ discrimination.

\section{DISCUSSION}

$\mathrm{T}$ his study was designed to examine the effect of presentation level on speech discrimination for infants with HL and to compare those effects to those observed for infants with $\mathrm{NH}$. We also sought to determine whether presentation level effects differ between speech contrasts believed to differ in discrimination difficulty. Our findings showed that both infants with HL and infants with $\mathrm{NH}$ would be expected to discriminate the /a-i/ contrast at 56 to $63 \mathrm{dBA}$. The presentation level at which most infants in either group could discriminate /ba-da/ could not be determined because many infants did not succeed at discriminating the /ba-da/ contrast. Finally, we found that for infants with HL aided SII and HFPTA were significantly related to success on /a-i/ discrimination, but not to success on /ba-da/ discrimination.

Infants with HL performed similarly to infants with $\mathrm{NH}$, as $95 \%$ of infants with HL and $86 \%$ of infants with $\mathrm{NH}$ were able to successfully discriminate /a-i/. Furthermore, there was not a significant difference between groups in the presentation level required to reach criterion. The only difference between infants with and without HL was that/ba-da/ seems to be more difficult for infants with HL compared with infants with NH relative to their performance on /a-i/. Although infants with HL and infants with NH need similar presentation levels to reach criterion on these two speech contrasts that is not to say that 
infants with $\mathrm{HL}$ and infants with $\mathrm{NH}$ require the same sensation level to discriminate speech sounds: The sound level in the ear canal for stimuli presented in soundfield would be expected to be greater for infants using HAs compared with NH infants because of amplification by the HA. To determine whether infants with HA and infants with $\mathrm{NH}$ differ in the sensation level required to discriminate between speech sounds, both aided thresholds and earcanal measures of stimulus level would be need to be measured concurrently in the booth during testing sessions.

Twenty-nine percent of infants with $\mathrm{NH}$ did not reach criterion performance for /ba-da/ at any level, consistent with the $28 \%$ of 6 - to 8-month-old infants who did not reach criterion on /ba-da/ discrimination reported by Nozza (1987). However, half the infants with HL did not reach criterion on /ba-da/ at any level. Although about the same proportion of infants with HL and infants with $\mathrm{NH}$ reached criterion at $50 \mathrm{dBA}$, infants with $\mathrm{HL}$ who did not reach criterion at $50 \mathrm{dBA}$ were less likely than those with $\mathrm{NH}$ to be helped by increases in presentation level. However, infants with HL who did not reach criterion on one or both contrasts did not statistically differ in age, gender, aided 4F PTA, or order of presentation from the infants who reached criterion. Finally, we considered hearing age and found that it was not a significant predictor of reaching criterion $(p=0.70)$. It is noteworthy that for infants with HL, audibility alone was found to be sufficient to predict /a-i/ discrimination, but was insufficient to predict discrimination for /ba-da/. Even for those with high levels of audibility, /ba-da/ discrimination was not guaranteed. Thus, the variables that predict successful /ba-da/ discrimination remain to be identified.

That fewer infants reached criterion on the /ba-da/ contrast is consistent with previous reports finding that vowels (/a-i/) are easier to discriminate than contrasts which vary in place of articulation (/ba-da/; reports) (Eilers et al, 1981; Boothroyd, 1984; Rosen, 1992; van Wieringen and Wouters, 1999; Martinez et al, 2008; Fredrickson, 2010; Uhler et al, 2011). This difference may be related to the fact that vowels and consonants may have different functionality in language acquisition and learning (Toro et al, 2008) and that language-specific vowel perception emerges earlier than that of consonants (Strange and Jenkins, 1978; Werker and Tees, 1984). The current findings, along with those of previous studies, suggest that further research into developmental differences between vowels and consonants is warranted, particularly for infants with HL.

Despite the fact that fewer infants reached criterion on /ba-da/, it is likely that the infants with $\mathrm{NH}$, in any case, were able to distinguish these two sounds. To interpret a failure to discriminate by an infant with HL, it would be necessary to show that nearly all infants with $\mathrm{NH}$ were successful at the discrimination under the same conditions. While increasing the presentation level beyond $70 \mathrm{dBA}$ might increase the proportion of normal-hearing infants reaching criterion, it is possible that increasing the level would not be helpful for infants who use HAs due to input compression. Changes in procedure, such as increasing the intensity of the target sound on initial trials to draw attention to the change, may also improve the success rate on /ba-da/. Ultimately, objective measures may be useful in evaluating the degree to which the HA or CI is providing an infant with sufficient information to support speech discrimination.

\section{Limitations}

Although few differences were observed in discrimination abilities between the infants with $\mathrm{HL}$ and the infants with $\mathrm{NH}$, the relatively small sample size is clearly a limitation in the interpretation of that finding. Thus, additional investigation is warranted to determine clinic norms for discrimination abilities at multiple presentation levels for children with various degrees of HL both with HAs and CIs. Although no relationship between /ba-da/ discrimination and SII was observed, it is possible that a lack of measured RECDs for every HA user reduced the accuracy of the SII calculation. Our future investigations will include such measures, so that we may more accurately translate these clinical findings back to the degree of audibility and access for the children wearing HAs. Finally, there were two children who did not condition and had to be excluded: one to the hearing test (bilateral CI user) and one to VRISD (bilateral HA user). This highlights the broader limitation of behavioral assessment methods and indicates that we need a task to assess hearing and speech perception in this group of children who are unable to be conditioned.

\section{CONCLUSIONS}

$\mathrm{M}$ ost infants with and without HL exhibited /a-i/ discrimination at a presentation level of 56-63 dBA. Infants with HL exhibited significantly greater discrimination abilities for /a-i/ as compared with /ba-da/, whereas infants with $\mathrm{NH}$ exhibited no differences in discrimination for the two pairs of contrasts. However, the presentation level at which most infants could discriminate /ba/ from /da/ could not be estimated with the current procedure. The findings of this study suggest that speech sounds should be presented at multiple intensity levels to ensure that a full assessment of an infant's speech discrimination abilities is obtained. Finally, audibility alone does not necessarily predict discrimination abilities, and therefore, discrimination tasks should be a part of pediatric minimum speech test batteries for validation of $\mathrm{HA}$ and CI fittings.

Acknowledgments. I would like to express my sincere appreciation of Dr. Randal G. Ross for his thoughtful and thorough review of this article. His contributions to science and mentorship will forever be remembered with sincere appreciation and 
gratitude. We would like to express our gratitude to all families, Colorado Home Intervention Program, and audiologists who participated in this project.

\section{REFERENCES}

Boothroyd A. (1984) Auditory perception of speech contrasts by subjects with sensorineural hearing loss. J Speech Hear Res 27(1):134-144.

Eilers RE, Morse PA, Gavin WJ, Oller DK. (1981) Discrimination of voice onset time in infancy. J Acoust Soc Am 70(4):955-965.

Eilers RE, Wilson WR, Moore JM. (1977) Developmental changes in speech discrimination in infants. J Speech Hear Res 20(4):766-780.

Fredrickson T. (2010) Visual Reinforcement Infant Speech Discrimination: Developing a Method of Performance Analysis.Unpublished Dissertation. University of Colorado - Boulder.

Gravel J. (1989) Behavioral assessment of auditory function. Semin Hear 10:216-228.

Harrison M, Roush J. (1996) Age of suspicion, identification, and intervention for infants and young children with hearing loss: a national study. Ear Hear 17(1):55-62.

Holte L, Walker E, Oleson J, Spratford M, Moeller MP, Roush P, Ou H, Tomblin JB. (2012) Factors influencing follow-up to newborn hearing screening for infants who are hard of hearing. Am J Audiol 21(2):163-174.

Martinez A, Eisenberg L, Boothroyd A, Visser-Dumont L. (2008) Assessing speech pattern contrast perception in infants: early results on VRASPAC. Otol Neurotol 29(2):183-188.

Macmillan NA, Creelman CD. (2005) Detection Theory: A User's Guide. Mahwah, NJ: Lawrence Erlbaum Associates, Inc.

McArdle R, Hnath-Chisolm T. (2009) Speech audiometry. In: Katz J, Medwetsky L, Burkard R, Hood L, eds. Handbook of Clinical Audiology. 6th ed. Baltimore, MD: Lippincott Williams and Wilkins.

McCreery RW, Bentler RA, Roush PA. (2013) Characteristics of hearing aid fittings in infants and young children. Ear Hear 34(6):701-710.

McCreery RW, Walker EA, Spratford M, Oleson J, Bentler R, Holte L, Roush P. (2015) Speech recognition and parent-ratings from auditory development questionnaires in children who are hard of hearing. Ear Hear 36(Suppl 1):60S-75S.

Moeller MP. (2000) Early intervention and language development in children who are deaf and hard of hearing. Pediatrics 106(3):E43.

Moeller MP, Hoover B, Peterson B, Stelmachowicz P. (2009) Consistency of hearing aid use in infants with early-identified hearing loss. Am J Audiol 18(1):14-23.

Nozza RJ. (1987) Infant speech-sound discrimination testing: effects of stimulus intensity and procedural model on measures of performance. J Acoust Soc Am 81(6):1928-1939.

Nozza RJ. (2000) Thresholds are not enough: understanding how infants' process. Speech has a role in how we manage hearing loss. In: Seewald RC, ed. A Sound Found through Early Amplification. Chicago, IL: Phonak AG.

Nozza RJ, Miller SL, Rossman RN, Bond LC. (1991) Reliability and validity of infant speech-sound discrimination-in-noise thresholds. J Speech Hear Res 34(3):643-650.
Nozza RJ, Wilson WR. (1984) Masked and unmasked pure-tone thresholds of infants and adults: development of auditory frequency selectivity and sensitivity. J Speech Hear Res 27(4):613-622.

Rosen S. (1992) Temporal information in speech: acoustic, auditory and linguistic aspects. Philos Trans R Soc Lond B Biol Sci 336(1278):367-373.

Scollie S, Seewald R, Cornelisse L, Moodie S, Bagatto M, Laurnagaray D, Beaulac S, Pumford J. (2005) The desired sensation level multistage input/output algorithm. Trends Amplif 9(4):159-197.

Stiles DJ, Bentler RA, McGregor KK. (2012) The speech intelligibility index and the pure-tone average as predictors of lexical ability in children fit with hearing AIDS. J Speech Lang Hear Res 55(3):764-778.

Strange W, Jenkins JJ. (1978) Role of linguistic experience in the perception of speech. In: Pick H, ed. Perception and Experience. New York, NY: Plenum, 125-169.

Tomblin JB, Oleson JJ, Ambrose SE, Walker E, Moeller MP. (2014) The influence of hearing aids on the speech and language development of children with hearing loss. JAMA Otolaryngol Head Neck Surg 140(5):403-409.

Tomblin JB, Walker EA, Mccreery R, Arenas MA, Harrison M, Moeller MP. (2015) Ear and hearing outcomes of children with hearing loss : data collection and methods. Ear Hear 36:14-23.

Toro JM, Shukla M, Nespor M, Endress AD. (2008) The quest for generalizations over consonants: asymmetries between consonants and vowels are not the by-product of acoustic differences. Percept Psychophys 70(8):1515-1525.

Tsao FM, Liu HM, Kuhl PK. (2004) Speech perception in infancy predicts language development in the second year of life: a longitudinal study. Child Dev 75(4):1067-1084.

Uhler K, Gifford RH. (2014) Current trends in pediatric cochlear implant candidate selection and postoperative follow-up. Am $J$ Audiol 23(3):309-325.

Uhler KM, Baca R, Dudas E, Fredrickson T. (2015) Refining stimulus parameters in assessing infant speech perception using visual reinforcement infant speech discrimination: sensation level. J Am Acad Audiol 26(10):807-814.

Uhler K, Yoshinaga-Itano C, Gabbard SA, Rothpletz AM, Jenkins H. (2011) Longitudinal infant speech perception in young cochlear implant users. J Am Acad Audiol 22(3):129-142.

Uhler K, Oropereza J, Awad R. (2016) Quality Improvement in Tracking JCIH Outcome Variables. All Staff, Aurora, CO.

van Wieringen A, Wouters J. (1999) Natural vowel and consonant recognition by Laura cochlear implantees. Ear Hear 20(2):89-103.

Walker EA, McCreery RW, Spratford M, Oleson JJ, Van Buren J, Bentler R, Roush P, Moeller MP. (2015) Trends and predictors of longitudinal hearing aid use for children who are hard of hearing. Ear Hear 36(Suppl 1):38S-47S.

Walker EA, Spratford M, Moeller MP, Oleson J, Ou H, Roush P, Jacobs S. (2013) Predictors of hearing aid use time in children with mild-to-severe hearing loss. Lang Speech Hear Serv Sch 44(1):73-88.

Werker RC, Tees JF. (1984) Cross-language speech perception: evidence for perceptual reorganization during the first year of life. Infant Behav Dev 7:49-63. 